\title{
HIGH SPEED PHOTOMETRY OF AM CVnS WITH THE UCT CCD
}

\author{
Patrick A. Woudt ${ }^{1}$ and Brian Warner ${ }^{1}$
}

\begin{abstract}
RESUMEN
Continuando con nuestro "survey" fotométrico de alta velocidad de variables cataclísmicas débiles, se han reconocido dos CVs como degeneradas dobles que transfieren helio; se trata de ES Cet $\left(P_{\text {orb }}=620.21144 \mathrm{~s}\right)$ y 2003aw $\left(P_{s h}=2041.5 \mathrm{~s}\right)$. Hemos observado ES Cet fotométricamente durante más de dos años para seguir la evolución del período orbital. Ofrecemos una explicación alternativa para la naturaleza de V407 Vul (que fue clasificada como una ultracompacta junto con RX J0806 y ES Cet), la cual elimina a V407 Vul de la clasificación AM CVn.
\end{abstract}

\section{ABSTRACT}

Following our high speed photometric survey of faint cataclysmic variables, two CVs were recognised to be helium-transferring double degenerates; these are ES Cet $\left(P_{\text {orb }}=620.21144 \mathrm{~s}\right)$ and 2003aw $\left(P_{s h}=2041.5 \mathrm{~s}\right)$. We have now observed ES Cet photometrically for over two years in order to follow the evolution of the orbital period. We offer an alternative explanation for the nature of V407 Vul (which was classified as an ultra-compact together with RX J0806 and ES Cet); this will take V407 Vul out of the AM CVn classification.

Key Words: BINARIES: CLOSE - NOVAE, CATACLYSMIC VARIABLES - STARS: INDIVIDUAL (ES CET, 2003AW) - TECHNIQUES: PHOTOMETRIC

\section{INTRODUCTION}

The AM CVn stars are double degenerate interacting binaries - i.e., they have the structure of cataclysmic variable stars, transferring mass from a donor through a stream and accretion disc to an accretor - but both stellar components are white dwarfs. Currently there are ten stars (ES Cet, AM CVn, HP Lib, CR Boo, KL Dra, V803 Cen, CP Eri, GP Com, CE-315), with orbital periods ranging from 10.3 to $65.1 \mathrm{~min}$, that are definitely of AM CVn type, and two possible candidates, RX J0806.3+1527 (Israel et al. 2002) and V407 Vul (Cropper et al. 1998, Marsh \& Steeghs 2002), with periods of 5.4 and 9.5 min, respectively. The interpretation of these as AM CVn systems has been questioned (Norton, Haswell \& Wynn 2002; Warner 2004). A new candidate AM CVn star has very recently been recognised (Roelofs et al. 2004, these proceedings). The general properties of AM CVn stars and their evolutionary status are reviewed in Warner (1995a,b); see also Nelemans, Yungelson \& Portegies Zwart (2004).

\section{AM CVN STARS IN THE UCT CCD CV SURVEY}

Two of the AM CVn stars listed in the introduction, were recognised as such during the UCT CCD CV Survey, namely ES Cet (Warner \& Woudt 2002)

\footnotetext{
${ }^{1}$ Department of Astronomy, University of Cape Town, Rondebosch 7700 , South Africa.
}

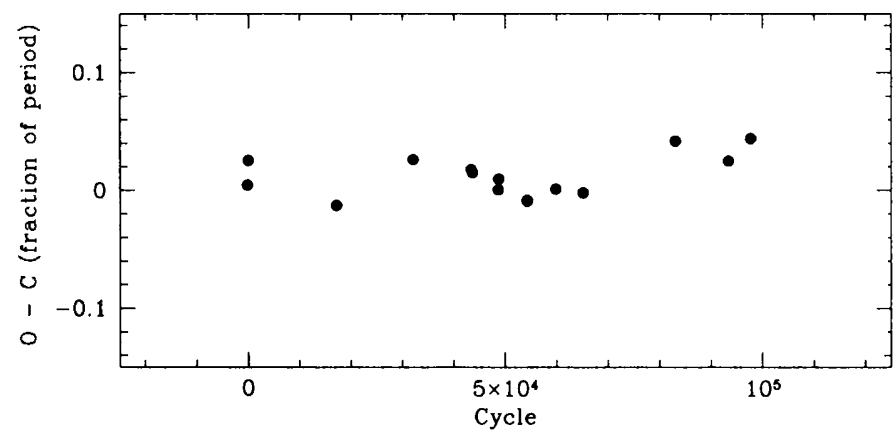

Fig. 1. The O-C diagram of all the UCT CCD photometry obtained of ES Cet over the last two years.

and '2003aw' (Woudt \& Warner 2003a). The latter was initially thought to be a supernova (WoodVasey et al. 2003), but spectroscopy of the supernova candidate revealed weak $\mathrm{He}$ I emission lines at zero redshift (Chornock \& Filippenko 2003), suggesting it might be an AM CVn star caught in a high state.

\subsection{ES Cet}

High speed photometry of ES Cet obtained in 2001 October during four nights (Warner \& Woudt 2002) showed a clear modulation at $620.26 \mathrm{~s}$. In the Fourier transform, only the fundamental and the first three harmonics of the 620.26-s modulation were present. The spectrum of ES Cet (Steeghs 2003; Woudt \& Warrer 2004) is dominated by helium emission lines.

Two years of photometry of ES Cet shows a very 
stable O-C diagram (see Fig. 1); there is some scatter, but no substantial phase shifts or period changes (as might have been expected were the modulation due to superhumps) are present.

With a two year baseline, there is a slight hint of upwards curvature in the $\mathrm{O}-\mathrm{C}$ diagram. This would imply a lengthening of the photometric period. This is confirmed by separating the data in two halves. The amount of $\dot{P}$ implied by the O-C diagram is $\sim 1.6 \times 10^{-11}$ (Woudt \& Warner 2004), close to the expected rate of change of $6 \times 10^{-12}$ (Warner \& Woudt 2002) for a system of high mass-transfer rate $\dot{M}$. Further observations over the next few years need to be made to confirm this suggestion.

\section{2. '2003aw'}

We have obtained high-speed photometry of '2003aw' during the high state of 2003 February and during its decline into the intermediate state (Woudt \& Warner 2003a). Both in the high state and in the intermediate state we found an identical photometric period (presumably a superhump period) of $P_{s h}=2041.5 \pm 0.3 \mathrm{~s}$, although the average light curve during the high state was very different from the mean light curve during the intermediate state. In the high state, recurrent dips were seen which initially were thought to be shallow eclipses (Woudt \& Warner 2003b). These dips were seen to repeat on the 2041-s modulation and can only be eclipses if the photometric period is the orbital period rather than the superhump period (in ES Cet the photometric modulation has also been suggested to be orbital in nature; Woudt \& Warner 2004). None of the AM CVn stars so far are eclipsing systems, even though $\gtrsim 30 \%$ of the systems at $P=1000 \mathrm{~s}$ are expected to be eclipsing systems (Nelemans et al. 2004).

'2003aw' fits very well in the emerging hierarchy of AM CVn stars (it strongly resembles CR Boo and V803 Cen); systems of shortest period ( $\lesssim 1200 \mathrm{~s}$ ) have stable high $\dot{M}$ discs, systems with periods in the range $\sim 1200-2500 \mathrm{~s}$ (to which '2003aw' belongs) have unstable high $\dot{M}$ discs, and systems with orbital periods $\gtrsim 2500$ s have low $\dot{M}$.

\section{3. $R X J 0806.3$}

Two recent papers (Hakala et al. 2003; Strohmayer 2003) reported the detection of a spinup in RX J0806.3. It is argued by Woudt \& Warner (2004) that there is insufficient data coverage to make such a claim with high accuracy. The reported period change depends critically on the ROSAT 1994/1995 data. Those data suffer from significant aliasing, and if the period is wrong by one cycle (in 50000 cycles) the trend completely disappears.

A detected period change will, however, have important implications for understanding what drives the period evolution (mass transfer, gravitational radiation) in this proposed ultra-short period system.

\section{4. $V 407 \mathrm{Vul}$}

Warner (2004), in a paper on intermediate polars in a low state, has recently proposed that given its similarities to V471 Tau, V407 Vul could be a long period pre-CV. Steeghs (2003) has obtained a spectrum of V407 Vul which shows it to be an apparently normal reddened $\mathrm{K}$ star, with the addition of a featureless pulsed component at shortest. wavelengths. There are no emission lines and there is no helium present; its credentials as an AM CVn system are doubtful.

PAW's research is funded by a strategic grant from the University of Cape Town and by funds from the National Research Foundation. BW's research is funded by the University of Cape Town.

\section{REFERENCES}

Chornock, R., \& Filippenko, A. V. 2003, IAUC, 8084. 3 Cropper, M., Harrop-Allin, M. K., Mason, K. O., Mittaz, J. P. D., Potter, S. B., \& Ramsay, G. 1998, MNRAS, 293, 57L

Hakala, P., Ramsay, G., Wu, K., et al. 2003, MNRAS. $343,10 \mathrm{~L}$

Israel, G.-L., Hummel, W., Covino, S., et al. 2002, A\&A. $386,13 \mathrm{~L}$

Marsh, T. R., \& Steeghs, D. 2002, MNRAS, 331, 7L

Nelemans, G., Yungelson, L. R., \& Portegies Zwart, S. F. 2004, MNRAS, in press (astro-ph/0312193)

Norton, A. J., Haswell, C. A., \& Wynn, G. A. 2002, astro$\mathrm{ph} / 0206013$

Steeghs, D. 2003, Workshop on Ultracompact Binaries. Santa Barbara.

Strohmayer, T. E. 2003, ApJ, 593, 39L

Warner, B. 1995a, Cataclysmic Variable Stars, Cambridge University Press, Cambridge

Warner, B. 1995b, Ap\&SS, 225, 249

Warner, B. 2004, in 'White Dwarfs: Galactic and Cosmological Probes', eds. E. Sion, S. Vennes and H. Shipman (astro-ph/0310243)

Warner, B., \& Woudt, P. A. 2002, PASP. 114, 129

Wood-Vasey, W. M., Aldering, G., Nugent. P.. \& Li, h 2003, IAUC, 8077, 1

Woudt, P. A., \& Warner, B. 2003a, MNRAS, 345. 1260

Woudt, P. A., \& Warner, B. 2003a, IAU Circ, s085, 3

Woudt, P. A., \& Warner, B. 2004, in 'White Dwarfs: Galactic and Cosmological Probes', eds. E. Sion, S Vennes and H. Shipman (astro-ph/0310494) 